\title{
Mutations on CaENO1 in Candida albicans inhibit cell growth in the presence of glucose
}

\author{
Yun-Liang Yang*, Hsing-Fang Chen, Tai-Jung Kuo \& Chi-Yang Lin \\ Department of Biological Science and Technology, National Chiao Tung University, Hsinchu, Taiwan, \\ Republic of China
}

(C) 2006 National Science Council, Taipei

Key words: Candida albicans, cell growth, enolase, glucose, inhibition

\begin{abstract}
Summary
Enolase (2-phospho-D-glycerate hydrolase) is an enzymatic component of the glycolytic pathway and is conserved through evolution. The TR-CaENOI/Caenol stain, of which the expression of CaENOI is under control of the tetracycline-regulatable (TR) expression system, is utilized for elucidating the functions of CaENO1 in Candida albicans. As expected, there was no detectable CaENO1 mRNA when the $T R$-CaENOI/Caenol cells grew on media containing doxycycline repressing the expression of $T R$ - $\mathrm{Ca}$ ENO1.The TR-CaENO1/Caenol cells were arrested in media containing doxycycline in the presence of glucose but not in non-fermentable carbon sources, such as glycerol. Furthermore, the TR-CaENOI/ Caenol cells were also arrested in media containing 4\% serum. In this study, we have showed that CaENO1 is required for the cell growth of C. albicans in the presence of glucose. Our findings may help us to design new and more effective antifungal agents for preventing and treating bloodstream fungal infections by blocking the function(s) of enolases.
\end{abstract}

\section{Introduction}

Enolase (2-phospho-D-glycerate hydrolase) is an enzymatic component of the glycolytic pathway, catalyzing the conversion of 2-phospho-D-glycerate to phosphoenolpyruvate. It is a highly conserved protein throughout the kingdoms of life from plants to animals and from bacteria to humans [1].

Enolase and other glycolytic enzymes are the most abundant proteins in Saccharomyces cerevisiae $[2,3]$. There are two non-tandemly repeated enolase structural genes, ENOI and ENO2, in the $S$. cerevisiae genome. These two genes share high degree of similarity within the coding sequences [4]. The amount of enzyme encoded by ENO1 increases

*To whom correspondence should be addressed. Phone: 8863-571-2121 ext. 56920; Fax: 886-3-572-9288; E-mail: yyang@ mail.nctu.edu.tw during stationary phase while the amount of enolase encoded by ENO2 increases when glucose is present in the growth media [5,6]. Unlike other genes involved in glycolytic pathway, single mutation on either ENO1 or ENO2 in S. cerevisiae are viable in the presence of glucose $[6,7]$.

In Candida albicans, the gene encoding enolase is named CaENO1, of which the Ca stands for C. albicans [8]. Enolase has been estimated to comprise $0.7 \%$ of the total proteins in the yeastform cells and $2 \%$ in the hyphae [9]. Antagonizing CaENO1 expression by antisense RNA causes reduction of the growth rate in C. albicans [10].

The tetracycline-regulatable (TR) expression system is a well-established system for gene expression among eukaryotic cells [11-13]. To investigate the functions of enolase in C. albicans, we have constructed a TR-CaENO1/Caenol $C$. albicans strain, of which one copy of the 
CaENO1 gene is replaced by the $A R G 4$ selective marker and the other copy is under control of the TR expression system [14]. In the present study, we have showed that, unlike ENO1 and ENO2 of $S$. cerevisiae, CaENOl is essential for cell growth in glucose-contained media.

\section{Materials and methods}

\section{Strains and media}

Candida albicans strains used in this study

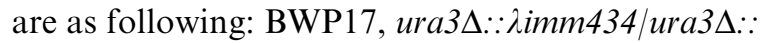
גimm434 his1::hisG/his1::hisG arg4::hisG/arg4::hisG [15], YLO139 (CaENO1/CaENO1), ura3s::

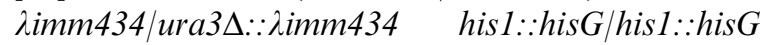
arg4::hisG/arg4::hisG CaENO1/tetR-HIS1-CaENO1 as the wild-type CaENO1 strain; YLO165

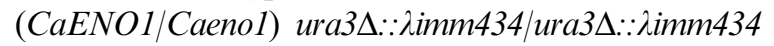
his1::hisG/his 1::hisG arg4::hisG/arg4::hisG CaENO/tetR-HIS1-Caeno1::ARG4 as the heterozygous mutant; and YLO166 (TR-CaENO1/Caenol),

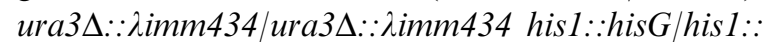
hisG arg4::hisG/arg4::hisG TR-CaENOI/ tetRHIS1-Caeno1::ARG4. All media described below were prepared as deletion in Sherman [16]: Basal medium (BASAL, 1\% Nutrient broth, 1\% mannitol, $0.2 \% \quad \mathrm{~K}_{2} \mathrm{HPO}_{4}$ ), Yeast Peptone Dextrose (YPD, 1\% yeast extract, 2\% peptone, and 2\% glucose), Yeast Peptone Glycerol (YPG, 1\% yeast extract, $2 \%$ peptone, and 2\% glycerol), Synthetic Dextrose (SD, $0.67 \%$ yeast nitrogen base without amino acid and $2 \%$ glucose), Brain Heart Infusion (BHI, 20\% infusion from calf brain, 15\% infusion from beef heart, $1 \%$ proteose pepton, $0.2 \%$ glucose, $0.5 \% \mathrm{NaCl}, 0.25 \% \mathrm{Na}_{2} \mathrm{PO}_{4}$ ), and Mueller Hinton ( $\mathrm{MH}, 0.03 \%$ beef extract, $1.75 \%$ Acid Hydrolysate of casein, and $0.015 \%$ starch). Equal volume of $2 \times$ media were mixed together to prepared for the mixed media. All agar plates were prepared with addition of $2 \%$ agar in media. Agar serum plates were prepared by mixing $1 \mathrm{ml}$ of serum into $25 \mathrm{ml}$ of $2 \%$ agar.

Construction of the CaENO1/Caenol heterozygous mutant

Candida albicans strain YLO139 (CaENO1/CaE$\mathrm{NO}$ ) was used for the construction of CaENO1/ Caenol heterozygous mutant (a gift from Dr. H.-J. Lo, NHRI, Taiwan). YLO139 is based on BWP17
[15] with the integration of the gene encoding the activator for the TR promoter, tetR, into the promoter of $\mathrm{CaENOI}$ alone with a His $1^{+}$marker.

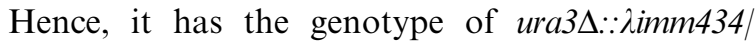

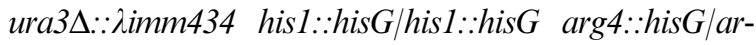
g4::hisG CaENO1/tetR-HIS1-CaENO1 (Figure 1) and the expression of tet $R$ was under the control of the CaENO1 promoter. To construct the CaENO1/Caenol heterozygous mutant, the plasmid pRS-ARG4 SpeI [15] was used as the template DNA. The primer ENO1KOF 5'd(ATGTCT TACG CCACTAAAAT CACGCCAGA TAC GTCTACG ACTCCAGAGG TTGGGTTGGCA ACTTCAAC GTTTTCCCAG CACGACGTT) and primer ENO1KOR 5'd(AGAGGCAAAC TTACAATTGA AAGCCTTTT GGAAATCT TT ACCAGCGTAG TAGCTTCAGAACCTAA TTC TGTGGAATTG TGAGCGGATA) were used to amplify a DNA fragment containing the $A R G 4$ gene sequence flanked by short homologous regions (70 bp) of the CaENO1 gene at the two extremities. This DNA fragment was then transformed into the YLO139 strain, containing the TR transactivator gene, tet $R$ [14] by the LioAC method. The open reading frame of CaENOI from position +1 to +1264 (The $A$ of the translation start codon ATG as +1 ) in the tet $R$ $H I S 1:: C a E N O 1$ allele was then replaced by the $A R G 4$ gene to generate the heterozygous mutant, YLO165 (CaENO1/Caenol), CaENO1/tetRHIS1-Caeno1::ARG4 (Figure 1). We named this mutant allele as Caenol.

Construction of the TR-CaENO1/Caenol strain

The primers ENO1AF 5'd(TTTGGTACCATTA AGCCGTGGGTTCTCA) and ENO1AR, 5'd(T TTCTCGAGCAATAAAGGGAGTAAGGTGG) were used to amplify a $C a E N O 1 A$ region from the 175 th to the 580th bp upstream of the translation initiation codon of the CaENOI gene. The primers ENO1BF, 5'd(GCTCTAGATGT CTTACGC CACTAAAATCC) and ENO1BR, 5'd(TTTCCG $C G G$ CAATGATGTCATTAACAT T) were used to amplify a $C a E N O 1 \mathrm{~B}$ region from the first to the 225 th $\mathrm{bp}$ downstream of translation initiation codon of the CaENO1 gene. These two regions were digested with restriction enzymes and ligated in the cloning regions of the p99CAU1 plasmid as described previously [14]. The strain YLO166 (TR-CaENO1/Caenol) (Figure 1) was generated 

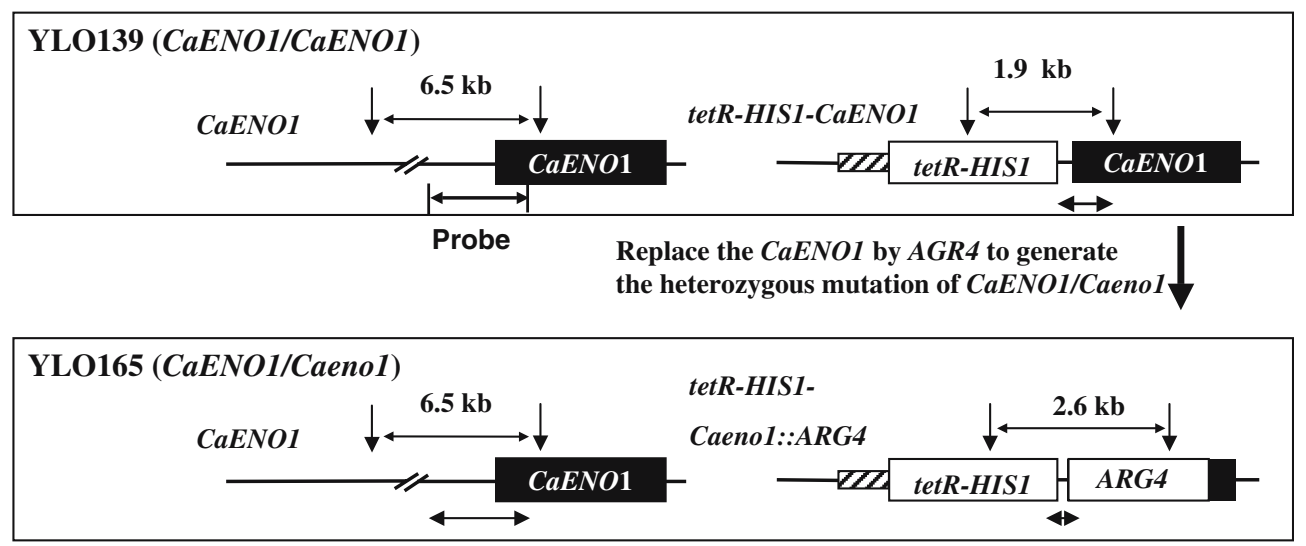

Insert TR promoter in front of CaENO1 such that

the expression of $\mathrm{CaENO1}$ is under control of the TR promoter

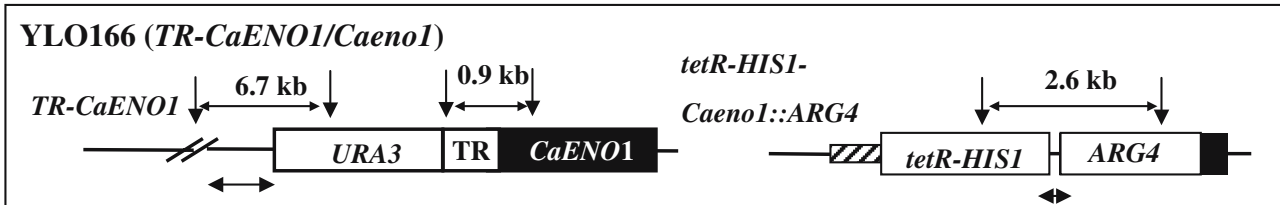

Figure 1. Scheme for the construction of the TR-CaENO1/Caeno1 strain. The C. albicans YLO139 strain contains the activator gene of TR, tetR in its genome alone with a His + marker (tetR-HISI) at the promoter of CaENOIto create the CaENOI/tetRHIS1-CaENO1 genotype. The open reading frame (solid bar) of CaENO1 in the tetR-HIS1-CaENOlallele was then replaced by the ARG4 gene (open bar) to generate the heterozygous mutant CaENO1/tetR-HIS1-Caeno1::ARG4, YLO165. Then, the TR promoter was inserted in front of the wild-type copy of CaENO1 to generateTR-CaENO1/tetR-HIS1-Caeno1::ARG4strain, YLO166. Thus, the expression of CaENO1 in the YLO166 strain was regulated by the TR expression system. Vertical arrows represent the restriction enzyme sites of $E c o$ RI. The predicated sizes (in $\mathrm{kb}$ ) of DNA fragments hybridized with the probe were indicated between two vertical arrows. The detecting regions of the probe are showed as double-headed arrows beneath the constructs. Probe, the region used for synthesized labeled DNA probe for Southern analysis.

by transforming the entire promoter-replacing construct into the YLO165 strain, such that the expression of CaENO1 is under control of the TR expression system.

\section{Southern blot analysis}

Total genomic DNA was isolated from each C. albicans strain and subjected to EcoRI digestion. The digested genomic DNA was then resolved in $0.7 \%$ agarose gel and transferred to a nylon membrane. The DNA fragment from 580th bp upstream to 205th bp downstream of the translation initiation codon of $C a E N O 1$ was used as the template to synthesize the DIG-labeled (Cat. No. 1175033. Roche, US) probes for Southern analysis. The experiment was performed according to the instruction from the manufacturer.

\section{Isolation of total RNA by hot acidic phenol}

The C. albicans cells were grown in $30 \mathrm{ml}$ of YPD liquid medium to the $\mathrm{OD}_{600}$ of 0.5 . The cells were then harvested after being incubated at $30^{\circ} \mathrm{C}$ for $1 \mathrm{~h}$ in the absence or presence of $20 \mu \mathrm{g} / \mathrm{ml}$ of doxycycline (Sigma D9891). The cell pellet was resuspended in RNA isolation buffer $(50 \mathrm{mM} \mathrm{NaO}$ Ac, $10 \mathrm{mM}$ EDTA, 1\% SDS). Acid-washed glass beads $(425-600 \mu \mathrm{m})$ and $500 \mu \mathrm{l}$ of $65^{\circ} \mathrm{C}$ acid phenol were added to the cell pellet. The cell suspension was vortexed vigorously for $10 \mathrm{~s}$ and then incubated at $65^{\circ} \mathrm{C}$ for $10 \mathrm{~min}$, followed by two extractions with acid phenol/chloroform and one extraction with chloroform. The RNA was then precipitated with ethanol and the quality determined by both the ratio of the absorption of $\mathrm{OD}_{260}-\mathrm{OD}_{280}$ and by electrophoresis in agarose gels. 
Northern blot analysis

Approximate $12 \mu \mathrm{g}$ of total RNA was resolved in a $1 \%$ agarose gel under denaturing conditions and then transferred to a nylon membrane. The CaE$N O 1 B$ region, from the first to the 225 th bp downstream of translation initiation codon of CaENO1, was used as the temple to generate a labeled probe for detection of $\mathrm{CaENO} 1$ expression by northern blot analysis using the DIG system (Cat. No. 1175033, Roche, US). The experiment was conducted according to the instruction supplied by the manufacturer.

\section{Growth assay on different media}

Both the wild-type strain, YLO139, and the TRCaENO1/Caenol strain, YLO166, were patched onto YPD agar medium without the doxycycline and then incubated at $30{ }^{\circ} \mathrm{C}$ for 1 day. Then they were replica-plated onto different agar media either in the absence or presence of $20 \mu \mathrm{g} / \mathrm{ml}$ doxycycline. All cells were incubated at $30{ }^{\circ} \mathrm{C}$ for 1 day. For the agar serum medium, cells were streaked onto agar plates containing 4\% serum and then incubated at $37^{\circ} \mathrm{C}$ for 2 days.

\section{Results and discussion}

\section{Construction of the TR-CaENO1/Caenol strain}

To utilize the TR expression system [14] for the study, we have obtained the YLO139 strain (Figure 1), a strain based on BWP17 with the integration of the gene encoding the trans-activator of the TR promoter, tet $R$, immediately downstream to the promoter of CaENO1. Then, the CaENO1 in the tetR-HIS1::CaENO1 allele was replaced by the $A R G 4$ gene to generate the heterozygous mutant (Figure 1, YLO165). Finally, the promoter region of the other copy of CaENOI was replaced by the TR promoter and hence under the TR system (Figure 1, YLO166). Doxycycline binds the tet $R$ trans-activator of the TR system and will prevent the interaction between the trans-activator and the $T R$ promoter [11-13]. As a consequence, in the presence of doxycycline, the gene expression from the $T R$ promoter is inhibited [14]. The genotypes of all constructed strains were verified by Southern analysis (Figure 2). In the YLO139 strain, the probe detected bands at the positions of 6.5 and $1.9 \mathrm{~kb}$. In the YLO165 heterozygous mutant strain, bands were at 6.5 and $2.6 \mathrm{~kb}$. And

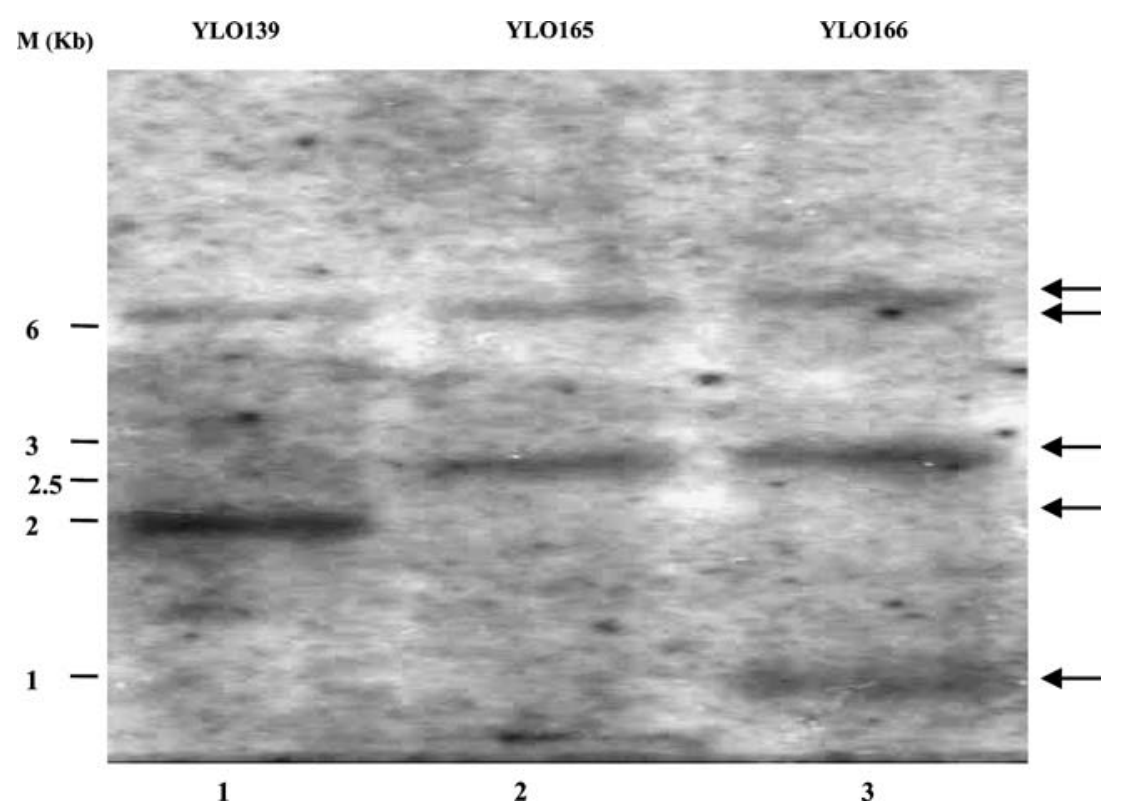

Figure 2. Result of Southern blot analysis of genomic CaENO1 in various strains. Genomic DNA digested with EcoRI was from the following strains: lane1, wild-type strain, CaENO1/CaENO1 (YLO139); lane2, heterozygous mutant, CaENO1/Caeno1(YLO165); lane 3, TR regulating CaENO1 strain, TR-CaENO1/Caeno1 (YLO166). The sizes (in kb) of the molecular weight standards are labeled on the left. The sizes of the bands (arrows on the right) were indicated in Figure 1 above each chromosomal construct between vertical arrows (the EcoRI restriction fragment). 


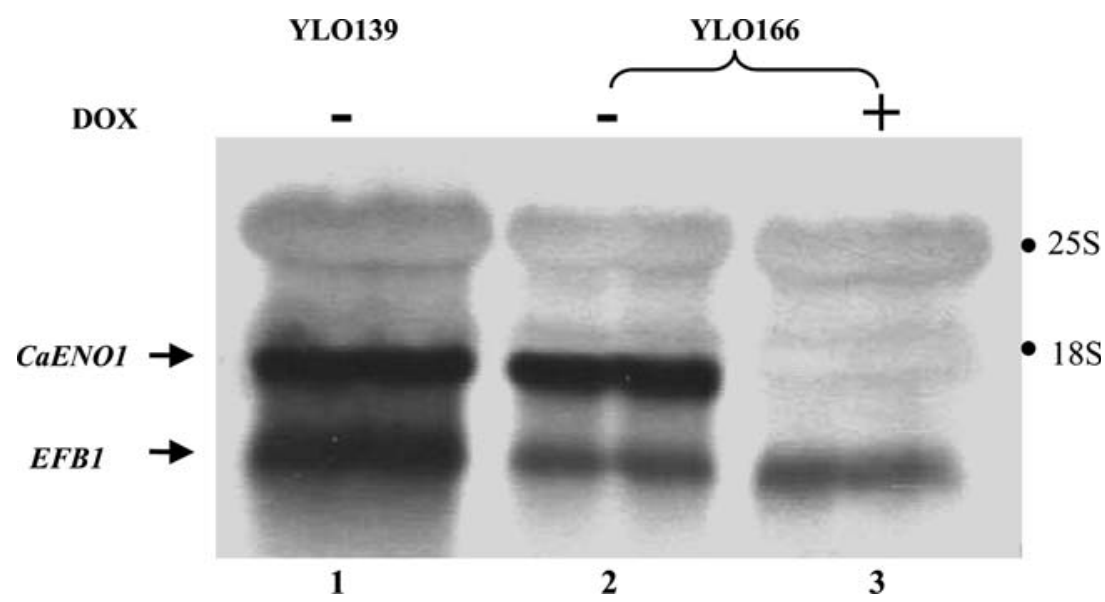

Figure 3. Northern blot analysis of the CaENO1 mRNA. Total RNA was isolated from the $C$. albicans strains for the analysis to assess the mRNA levels. (1), wild type (YLO139); (2), Caeno1/TR-CaENO1 (YLO166) without doxycycline; (3), TR-CaENO1/Caenol(YLO166) with doxycycline. The EFB1 gene was used as the loading control. The size of the mRNA for CaENO1 is about $1.5 \mathrm{~kb}$ and the loading control EFBl $0.7 \mathrm{~kb}$. DOX +, the medium containing $20 \mu \mathrm{g} / \mathrm{ml}$ of doxycycline; DOX -, in the absence of doxycycline.

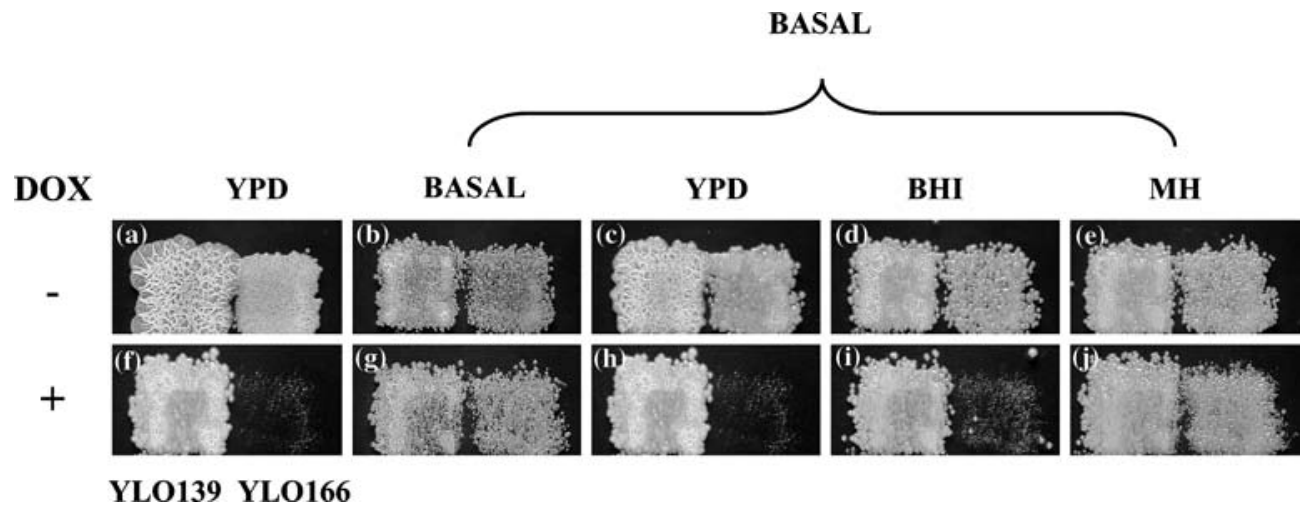

Figure 4. Cell growth on different media. The wild-type strain, YLO139 and the TR-CaENO1/Caeno1 strain, YLO166 were patched onto the YPD media and then cells were replica-plated onto different media after incubation at $30{ }^{\circ} \mathrm{C}$ for 1 day. The photographs were taken after the replica were incubated at $30^{\circ} \mathrm{C}$ for 1 day. The cells were grown on media in the absence of doxycycline (a-e) and in the presence of $20 \mu \mathrm{g} / \mathrm{ml}$ of doxycycline ( $\mathrm{f}-\mathrm{j}$ ). Media included YPD (a and $\mathrm{f}$ ), BASAL (b and g), BASAL plus YPD (c and h), BHI (d and i), and MH (e and j).

in the YLO166 strain, three bands positioned at 6.7, 2.6, and $0.9 \mathrm{~kb}$. The results of Southern analysis were as expected.

To elucidate the functions of enolase, we have to be able to turn off the expression of the CaENO1 in the YLO166 cells. Thus, to assess the expression of the gene in different strains, we applied Northern analysis to determine the mRNA level of the CaENO1. The level of CaENO1 mRNA of TR-CaENO1/Caenol YLO166 strain was determined after this strain was grown on media in the absence and presence of doxycycline. As expected, CaENO1 was expressed in the
YLO166 strain grown in the absence of doxycycline (Figure 3, lane 2); whereas, there was no detectable CaENOI mRNA when the strain was grown in the medium containing $20 \mu \mathrm{g} / \mathrm{ml}$ of doxycycline (Figure 3, lane 3). These results suggest that the expression of CaENOI in the YLO166 strain is indeed tightly regulated by the TR expression system.

\section{Investigation of cell growth on different media}

Both the wild-type, YLO139, and the TR-CaENO1/Caeno1, YLO166, were grown on different 


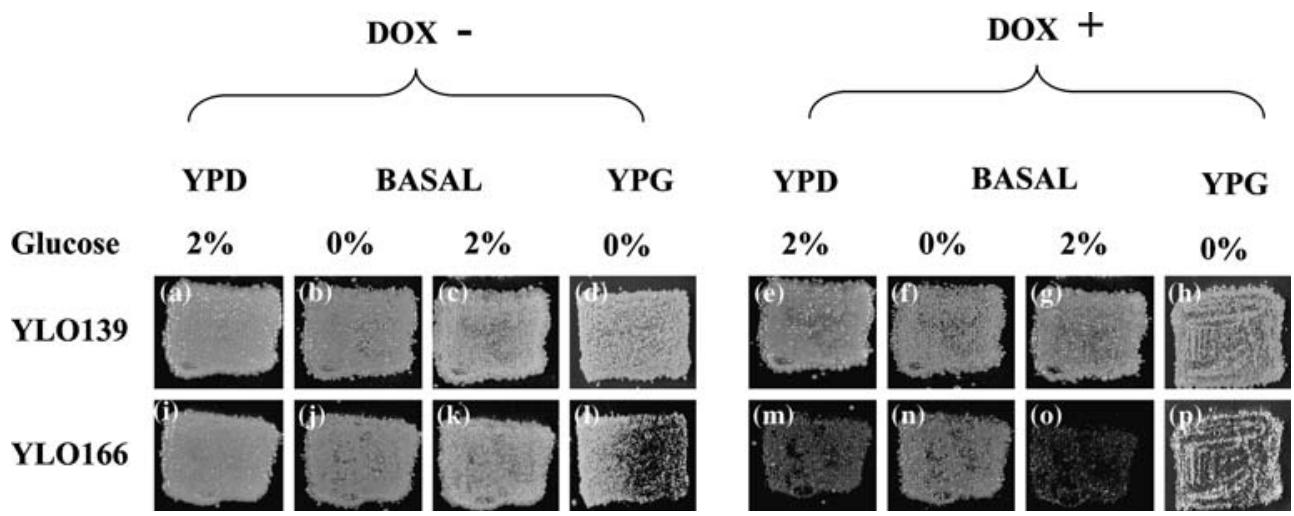

Figure 5. Enolase is required for cell growth in the presence of glucose. The wild-type strain, YLO139 (a-h) and the TR-CaENO1/ Caeno1 strain, YLO166 (i-p) were patched onto the YPD media and then cells were replica-plated onto different media after incubation at $3{ }^{\circ} 0 \mathrm{C}$ for 1 day. The photographs were taken after the replica were incubated at $30{ }^{\circ} \mathrm{C}$ for 1 day. The cells were grown on media in the absence of doxycycline (a-d and $\mathrm{i}-1)$ and in the presence of $20 \mu \mathrm{g} / \mathrm{ml}$ of doxycycline (e $-\mathrm{h}$ and $\mathrm{m}-\mathrm{p}$ ). Media included YPD plus $2 \%$ glucose (a, i, e, and m), BASAL (b, j, f, and n), BASAL plus $2 \%$ glucose (c, k, g, and o), and YPG (d, l, h, and p). DOX +, the medium containing $20 \mu \mathrm{g} / \mathrm{ml}$ of doxycycline; DOX-, in the absence of doxycycline.

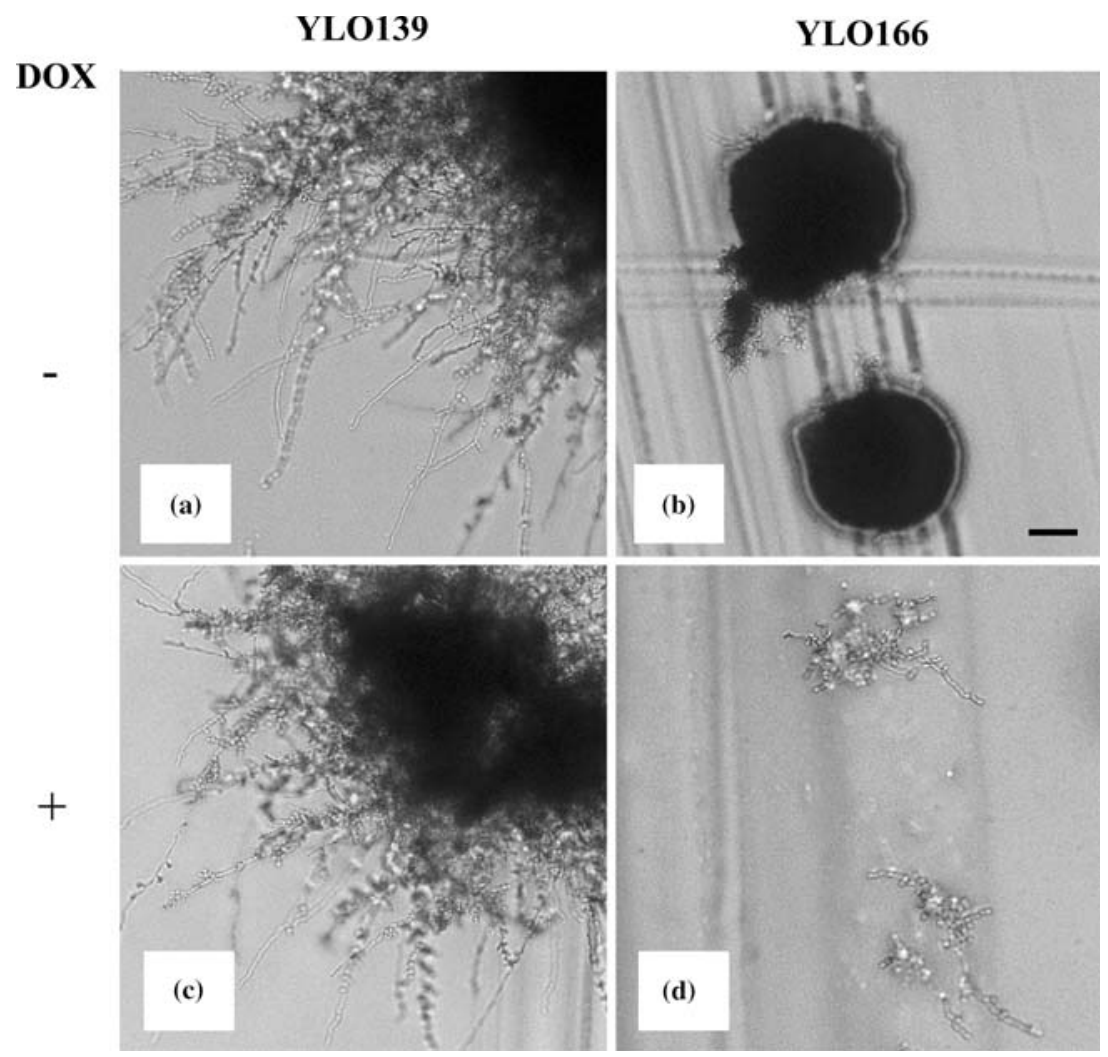

Figure 6. Cell growth on agar serum medium. The wild-type strain, YLO139 (a and c) and the TR-CaENO1/Caeno1 strain, YLO166 ( $\mathrm{b}$ and $\mathrm{d}$ ) were streaked on agar plates containing $4 \%$ serum media. The photographs were taken after incubation at $37^{\circ} \mathrm{C}$ for 2 days. The cells were grown on media in the absence of doxycycline ( $\mathrm{a}$ and $\mathrm{b}$ ) and in the presence of $20 \mu \mathrm{g} / \mathrm{ml}$ of doxycycline (c and d). Scale bar is $30 \mu \mathrm{m}$.

media to determine the functions of enolase in C. albicans (Figure 4). The wild-type strain grew on all media tested. And the YLO166 strain also grew well on all media in the absence of doxycycline. Interestingly, in the presence of doxycycline, the YLO166 strain grew on BASAL (Figure 4g) 
(a)

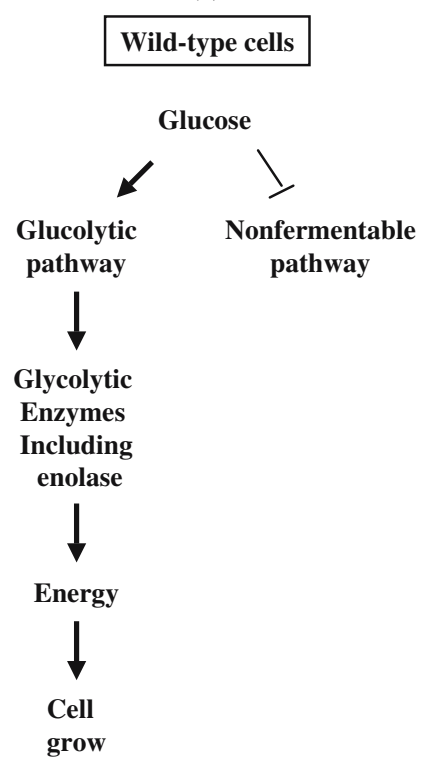

(b)

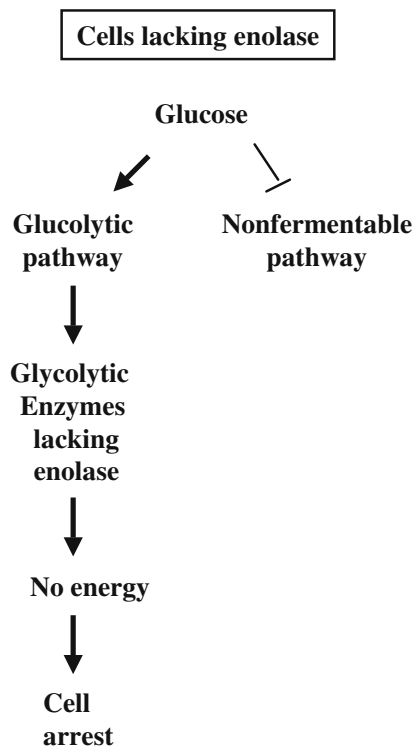

(c)

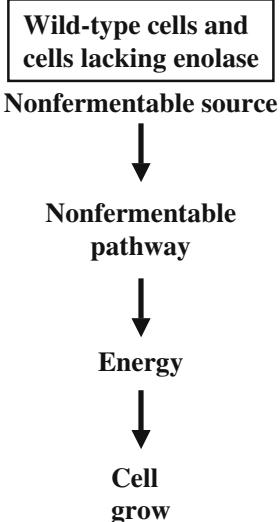

Figure 7. The schematic model for causing the arrest of cell growth by glucose repression. (a) The wild-type cells grow in media containing glucose. (b) Cells lacking enolase are arrested in media containing glucose. (c) Both the wild-type cells and cells lacking enolases grow in media containing only non-fermentable carbon sources.

but on YPD there was only a layer of arrested cells (Figure 4f). There are at least two explanations for this result. One is that the BASAL has positive factors required for the growth of cells lacking enolase. The other is that YPD has negative factors inhibiting the growth of cells not having enolase. To distinguish between these two possibilities, the YLO166 cells were grown on media with different combinations. The result showed that in the presence of doxycycline, cells grew on BASAL mixed with MH (Figure 4j) but not on BASAL with YPD (Figure 4h) or BHI (Figure 4i). One difference among these media is that both BASAL and MH do not contain glucose, whereas YPD and BHI do. Worthy mentioning is that BHI contains only $0.2 \%$ glucose. To determine if glucose is the factor affecting the growth of cells lacking enolase, we have patched the $T R-C a$ ENO1/Caeno1 YLO166 cells on BASAL plus 2\% glucose (Figure 5c, k, g, and o). Again, the wildtype cells grew on all media tested. In the presence of doxycycline, the YLO166 cells grew on BASAL medium in the absence of glucose (Figure $5 \mathrm{n}$ ) but not on BASAL medium with $2 \%$ glucose (Figure 5o). As showed in the Figure 5o, there was only a layer of arrested cells. To further assess our hypothesis that glucose inhibits the growth of cells lacking enolase, we have grown the $T R$-CaENO1/Caenol YLO166 cells on YPD and YPG media. Both the wild-type and the YLO166 cells grew on YPD and YPG in the absence of doxycycline (Figure 5a, i, d, and 1). In the presence of doxycycline, the YLO166 cells could grow on the YPG medium (Figure 5p) but on the YPD medium there was only a layer of arrested cells (Figure 5m). The only difference between the YPD and the YPG is that the former has glucose and the later has glycerol as the carbon source. Therefore, we concluded that enolase is required for the cell growth of C. albicans in media containing glucose. This datum also suggests that $S$. cerevisiae cells containing single mutations on either $E N O 1$ or $E N O 2$ are viable in the presence of glucose $[6,7]$ may have resulted from the functional redundancy of these two isoforms.

Our findings may help us to design new and more effective antifungal agents for preventing and treating bloodstream fungal infection by blocking the functions of enolase since serum also contain glucose. To investigate if this is a possibility, we have streaked $C$. albicans cells on agar serum plates. Again, the wild-type cells (Figure 6c) but not cells lacking enolase (Figure 6d) grew on media containing serum. 
Glucose is the most abundant monosaccharide in nature and it is also the primary fuel for yeasts. Although glucose and other sugars are the preferred carbon source by the yeast, non-fermentable substrates such as ethanol and glycerol can also be used for generating energy and cellular biomass [17-20]. Several regulatory networks of glucose repression are involved in coordinating the biosynthesis of enzymes required for the utilization of non-fermentable substrates [18, 21-24]. Thus, although yeasts can utilize various carbon sources, glucose-signaling pathways in yeasts ensure that they use up all the available glucose before turning toward alternative fuels (Figure 7a). When media contain only non-fermentable carbon sources, both the wild-type cells and the cells lacking enolase can utilize these sources to produce energy (Figure 7c). For the wild-type cells, glycolytic enzymes including enolase are important for generating energy while utilizing glucose. Therefore, cells lacking enolase cannot grow in media containing glucose because they are not able to utilize glucose. Neither can they utilize the non-fermentable carbon sources since genes involved in non-fermentable pathways are repressed by the presence of glucose (Figure 7b), which is consistent with the results in Figures 4 and 5.

In this study we have demonstrated that CaENO1 is essential for the $C$. albicans cells to grow on glucose. In the presence of glucose, lacking the CaENO1 gene became a limiting factor for cell growth due to glucose (catabolite) suppression. This also suggests that there is no other functional homolog of CaENO1 in C. albicans, which is different from that of $S$. cerevisiae. Finding genes whose products affect the cell growth of $C$. albicans in the presence of glucose may help to design new and more effective antifungal agents for preventing and treating bloodstream fungal infection since serum also contains glucose. It may be a possibility to reduce or block infections of $C$. albicans by using compounds that suppress CaENO1 activity.

\section{Acknowledgements}

We would like to thank Dr. H.-J. Lo and Dr. C.-G. Chen for their helps and the C. albicans strains YLO139. We would also like to thank Dr. H. Nakayama for the TR expression system. This work was supported in part by the Brain Research Center, National Chiao Tung University, University System of Taiwan, under grant 92B-711, and also in part by National Science Council under grants 94-2320-B-009-001.

\section{References}

1. Van der S.D., Rodrigues-Pousada R.A., Goodman H.M. and Van Montagu M., Plant enolase: gene structure, expression, and evolution. Plant Cell 3: 719-735, 1991.

2. Fauchon M., Lagniel G., Aude J.C., Lombardia L., Soularue P., Petat C., Marguerie G., Sentenac A., Werner M. and Labarre J., Sulfur sparing in the yeast proteome in response to sulfur demand. Mol. Cell 9: 713-723, 2002.

3. Kang J.J., Watson R.M., Fisher M.E., Higuchi R., Gelfand D.H. and Holland M.J., Transcript quantitation in total yeast cellular RNA using kinetic PCR. Nucleic Acids Res. 28: e2, 2000.

4. Holland M.J., Holland J.P., Thill G.P. and Jackson K.A., The primary structures of two yeast enolase genes. Homology between the $5^{\prime}$ noncoding flanking regions of yeast enolase and glyceraldehyde-3-phosphate dehydrogenase genes. J. Biol. Chem. 256: 1385-1395, 1981.

5. Iida H. and Yahara I., A heat shock-resistant mutant of Saccharomyces cerevisiae shows constitutive synthesis of two heat shock proteins and altered growth. J. Cell. Biol. 99: 1441-1450, 1984.

6. McAlister L. and Holland M.J., Targeted deletion of a yeast enolase structural gene. Identification and isolation of yeast enolase isozymes. J. Biol. Chem. 257: 7181-7188, 1982.

7. Niedenthal R., Riles L., Guldener U., Klein S., Johnston M. and Hegemann J.H., Systematic analysis of S. cerevisiae chromosome VIII genes. Yeast 15: 1775-1796, 1999.

8. Sundstrom P. and Aliaga G.R., Molecular cloning of cDNA and analysis of protein secondary structure of Candida albicans enolase, an abundant, immunodominant glycolytic enzyme. J. Bacteriol. 174: 6789-6799, 1992.

9. Sundstrom P. and Aliaga G.R., A subset of proteins found in culture supernatants ofCandida albicans includes the abundant, immunodominant, glycolytic enzyme enolase. J. Infect. Dis. 169: 452-456, 1994.

10. De Backer M.D., Nelissen B., Logghe M., Viaene J., Loonen I., Vandoninck S., de Hoogt R., Dewaele S., Simons F.A., Verhasselt P., Vanhoof G., Contreras R. and Luyten W.H., An antisense-based functional genomics approach for identification of genes critical for growth of Candida albicans. Nat. Biotechnol. 19: 235-241, 2001.

11. Baron U. and Bujard H., Tet repressor-based system for regulated gene expression in eukaryotic cells: principles and advances. Methods Enzymol. 327: 401-421, 2000.

12. Baron U., Gossen M. and Bujard H., Tetracycline-controlled transcription in eukaryotes: novel transactivators with graded transactivation potential. Nucleic Acids Res. 25: 2723-2729, 1997.

13. Tremblay P., Meiner Z., Galou M., Heinrich C., Petromilli C., Lisse T., Cayetano J., Torchia M., Mobley W., Bujard H., De Armond S.J. and Prusiner S.B., Doxycycline control of prion protein transgene expression modulates prion 
disease in mice. Proc. Natl. Acad. Sci. USA 95: 1258012585,1998

14. Nakayama H., Mio T., Nagahashi S., Kokado M., Arisawa M. and Aoki Y., Tetracycline-regulatable system to tightly control gene expression in the pathogenic fungus Candida albicans. Infect. Immun. 68: 6712-6719, 2000.

15. Wilson R.B., Davis D. and Mitchell A.P., Rapid hypothesis testing with Candida albicans through gene disruption with short homology regions. J. Bacteriol. 181: 1868-1874, 1999.

16. Sherman F., Getting started with yeast. Methods Enzymol. 350: 3-41, 2002

17. Carlson M. and Botstein D., Two differentially regulated mRNAs with different $5^{\prime}$ ends encode secreted with intracellular forms of yeast invertase. Cell 28: 145-154, 1982.

18. Johnston M., Feasting, fasting and fermenting. Glucose sensing in yeast and other cells. Trends Genet. 15: 29-33, 1999.

19. Rolland F., Winderickx J. and Thevelein J.M., Glucosesensing and -signalling mechanisms in yeast. FEMS Yeast Res. 2: 183-201, 2002.
20. Schuller H.J., Transcriptional control of nonfermentative metabolism in the yeast Saccharomyces cerevisiae. Curr. Genet. 43: 139-160, 2003.

21. Cereghino G.P. and Scheffler I.E., Genetic analysis of glucose regulation in saccharomyces cerevisiae: control of transcription versus mRNA turnover. EMBO J. 15: 363374, 1996.

22. Jiang H., Medintz I. and Michels C.A., Two glucose sensing/signaling pathways stimulate glucose-induced inactivation of maltose permease in Saccharomyces. Mol. Biol. Cell 8: 1293-1304, 1997.

23. Ullmann A., Catabolite repression: a story without end. Res. Microbiol. 147: 455-458, 1996.

24. Vallari R.C., Cook W.J., Audino D.C., Morgan M.J., Jensen D.E., Laudano A.P. and Denis C.L., Glucose repression of the yeast $A D H 2$ gene occurs through multiple mechanisms, including control of the protein synthesis of its transcriptional activator, ADR1. Mol. Cell. Biol. 12: 1663-1673, 1992. 